\title{
Traffic signal timing control using deep learning
}

\author{
Vishnu", Vikram, Jayakanthan, Likhita and Paavai Anand \\ SRM Institute of Science and Technology, Vadapalani, Chennai, India
}

Received: 21-November-2019; Revised: 21-December-2019; Accepted: 24-December-2019

(C)2019 Vishnu, Vikram et al. This is an open access article distributed under the Creative Commons Attribution (CC BY) License, which permits unrestricted use, distribution, and reproduction in any medium, provided the original work is properly cited.

\begin{abstract}
India is the second most populated country with 1.37 billion people so that avoiding traffic is impossible. But with proper traffic signal control method, we can control the amount of time spent in traffic. Our solution for this problem is to control the traffic signal timing and allocate more time length of green light to lanes containing a greater number of vehicles using deep learning-based computer vision approaches such as object detection. In January 2019, more than a million and a half $(1,607,315)$ new vehicles were bought and registered all across the country. In which $74 \%$ of the vehicles were two-wheelers and more than $80 \%$ of the total vehicles were petrol driven. India has 5.5 million kilometres of road network while now the number of vehicles registered is three times greater. These single statistics should reveal why Indian roads are getting more congested every month. In 2017, a total of 4,64,910 road accidents have been reported in which 1,47,913 deaths occurred and 4,70,975 people were injured. An average of 1274 accidents and 405 deaths every day. By using deep learning for controlling traffic signals, we can clear traffic more effectively and reduce traffic congestion, traffic violations, accidents, fuel consumption, pollution and time in traffic.
\end{abstract}

\section{Keywords}

Deep learning, Computer vision, Object detection, YOLO v3.

\section{Introduction}

Traffic congestion has caused many serious problems: time wastage, fuel wastage, air pollution, violation of traffic rules and accidents in and around the world. To prevent these problems, we need to find a productive way to reduce traffic congestion. The easiest and reliable way is to change the working method of traffic signal control. Traffic signal control provides a fixed time for each signal. In this method, the traffic signal does not work according to the realtime traffic, to elaborate, consider a four-way junction road where the traffic signal works on a fixed time basis. Now assume a single lane or two lanes containing more traffic compared to other lanes. Since the traffic signal works on a fixed time, the lanes with more traffic continue to increase and therefore this results in traffic congestion. This is because our traffic signals cannot fulfil the traffic demand without monitoring our traffic. To overcome this problem adaptive traffic signal control which adjusts signal timing according to real-time traffic by using object detection seems to be more efficient.

*Author for correspondence

27
For example, Zaidi et al. [1] proposed adaptive traffic signal control algorithms based on back-pressure method, which is similar to pushing water (here vehicles) to flow through a network of pipes(roads) by pressure gradients (the number of queued vehicles). Ghane et al. [2] proposed a method using q-learning and neural networks to find the number of vehicles present in each lane. Gao proposed a deep reinforcement learning algorithm that automatically extracts all features useful for adaptive traffic signal control from raw real-time traffic data.

In this paper, we propose an object detection method using machine learning based on the python programming language. We use OpenCV, NumPy, TensorFlow and other packages for library functions and time module is used to perform operations regarding time. Now we place the video camera containing the ability to detect vehicles near every signal, with the help of these cameras we can count the number of vehicles present in each lane in a fourway junction road. Now by comparing the number of vehicles in each lane, we can predict a lane which has more vehicles and therefore it has more traffic. The video cameras are connected with the traffic signals and we write an algorithm which makes the traffic 
signal to give more time for green signal in the lanes containing more traffic. Thus, reducing traffic congestion. We also take care such that the other lanes don't suffer from the starvation condition.

The objective of our model is to reduce the traffic rate with the given time limit. To avoid accidents and traffic violations. Last but not least to develop our methods suitable for the modern era.

\section{Literature survey}

In this section we explore other published papers in reducing traffic congestion.

Ghane, et al. [2] a paper on signal control using AI and machine learning used PARAMICS, a traffic simulator and tested it with various machine learning techniques to observe which was the best among them. They experimented with reinforcement learning (q-learning method), traditional networks and neural networks. Through observations it is concluded that q-learning method is more efficient compared to traditional networks but less efficient compared to neural networks. This is because neural networks provide the highest efficiency in terms of time delay. Another problem with q-learning method is that it only focuses on one junction. Therefore, even though it reduces traffic in one road but it fails to monitor other adjacent lanes. From this paper neural networks are more efficient to control traffic signal according to real time.

Zhang, et at. [3] proposed an intelligent traffic signal control using reinforcement learning with partial detection. They proposed a system based on partial detection of Dedicated Short-Range Communications (DSRC). They propose a transition scheme to Virtual Traffic Lights (VLT) which will reduce the commute time in traffic. The problem with this method is it won't work unless all vehicles have DSRC in them. The DSRC is implemented in United States but this type of vehicles is not operated in India. Therefore, this method does help with the traffic control issue we are facing.

Wei, et al. [4] proposed a deep learning method for traffic light control. They use deep q-learning method to remove traffic congestions in four junction road. Q-learning method is based on reward and punishment concept. They compared their methods with various other traffic light control methods. They tested their model on SUMO (simulation of urban mobility) a simulation platform. This method is complicated in multiple intersections but it controls the traffic light according to the real time traffic.

Liang, et al. [5] proposed the idea to implement reinforcement learning to control the traffic light according to the real time traffic. The reinforcement learning contains 3 states: the environment, the agent and a reward for every action. ALPHAGO is said to an example of reinforcement learning. ALPHAGO is computer program to play the game GO. They used Markov decision process, deep neural networks, and DQN (Deep Q-Network) methods to train their model.

Gao, et el. [6] proposed an adaptive traffic signal control using reinforcement learning and target network. Basically, they use conventional neural networks to make the model extract all the raw traffic data available in the environment and give a suitable output. The problem with reinforcement algorithm is that it is unstable or diverges in decision making they used experience relay and target network to simulate their model and train it. After comparison with other to popular algorithms, longest first queue algorithm and fixed time control algorithm, the results show that their model reduce the time delay in traffic and the stability of the model is maintained.

Dheekonda, et al. [7] proposed an idea of object detection in an advanced driver assistant system (ADAS) in automotive vehicles. By using conventional neural networks, they were able to classify the object in the environment. They used a camera of 8 megapixel and $77 \mathrm{GHZ}$ radar. The radar detects objects ahead, at the side and the rear for long and mid - range. The main idea is to improve robustness and accuracy in object detection using multi sensor and deep learning. They tested their model downloading various images for detecting objects. To detect object, they used ultrasonic distance sensor and sensor data from LIDAR/RADAR.

Li, et al. [8] proposed target detection of electronic components on PCB based on vision is the core technology for computer, communication and consumer electronics manufacturing companies to achieve quality control by using YOLO v3 algorithm to detect PCB's and classify accordingly. This algorithm's output layer that is sensitive to small targets and validates effectiveness of in a real PCB and virtual $\mathrm{PCB}$ picture test including a large number of PCB electronic components. 
Zhao, et al. [9] reviewed about deep learning-based object detection frameworks and its features. This review concludes that to gain complete image understanding, we should not only concentrate on classifying different images, but also try to precisely estimate the concepts and locations of objects contained in each image by this we have to choose the framework which suits better for performing object detection.

2.1 Abbreviations and acronyms YOLO v3- You Only Look Once Version 3

MATLAB - Matrix Laboratory

COCO- Common Objects in Context

CCTV- Closed Circuit Television

CSV-Comma-Separated Values

GUI- Graphical User Interface

\section{Methodology}

In this section we will provide a description on how our system works. To start, we use the python programming language to perform image processing using Yolo v3. It is the real-time object detection algorithm and a convolutional network which is used to perform object detection and localization of one or more objects within an image. The video footage is processed and fed to our object detection model frame by frame. Basic image processing techniques such as median filtering for noise removal and histogram equalization are performed to improve image quality. The object detection model localizes any vehicle in that frame and detects the vehicle. A variable keeping track of the number of vehicles is incremented every time a vehicle is detected. This approach can be used to measure the flow rate of traffic in multiple lanes and multiple directions.

The video footage of 4 different roads is taken by camera and each video footage is given as input one by one. The noises in the video is being removed and the image quality is being improved before converting it into frames. Each video is split into 60 frames per second and once the whole video is fully converted into frames, all the vehicles in each frame are being detected one by one. Each video will have an individual counter and the count will be incremented according to the vehicles that are detected. After the counter finishes counting, those counter values are saved in a CSV file. The csv file is then sent to the Matlab GUI program and the program compares each of the counter values and decides which counter value is maximum. And extra go time is being allocated for the road with maximum counter value by reducing the go time from other roads with lesser counter values and traffic will be simulated for one traffic cycle. After the timing are allocated the counters are reset and the counters start counting from the start and new timings are allocated. This program can be started after every traffic cycle since the traffic density in each lane constantly change with respect to time.

For example: We consider a junction with four roads, the green signal remains for 60 seconds in one lane, while the other lanes remain in red signal. The cycle continues in every lane so making a total time of 240 seconds. Now by our design, if there is more traffic on one particular road, there will be an increase of the green signal time for an extra 9 seconds thus the lane gets 69 seconds (extra 9 seconds is taken from other 3 lanes) and other lanes will get 57 seconds each (3 seconds from each lane is taken and made it as 9 seconds and this time is given to the road which have more traffic) so that the total time i.e., 240 seconds for one traffic signal cycle (after allowing vehicles from all the lanes for one time) will be same. Figures 1 and 2 shows the vehicle detection.

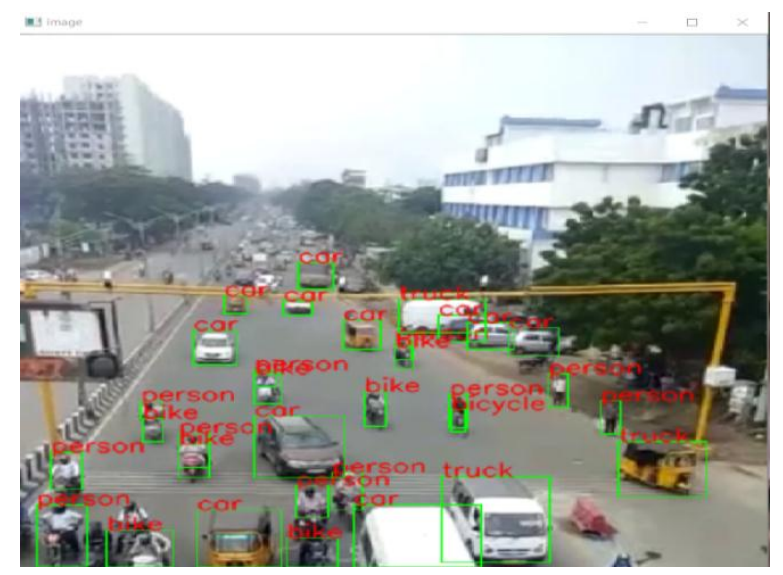

Figure 1 Vehicle detection - 1

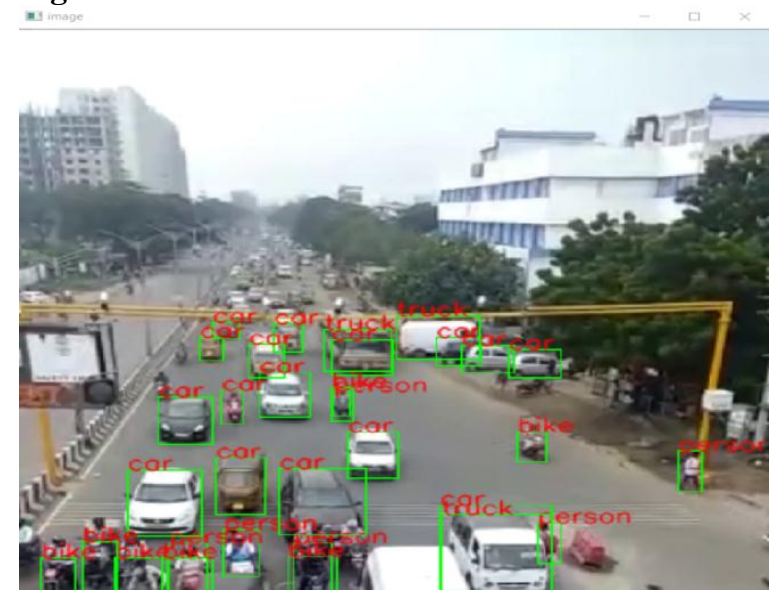

Figure 2 Vehicle detection - 2 
Vishnu, Vikram et al.

Figure 3 and 4 shows the traffic simulation. Figure 5 shows the working flowchart. Table 1 shows the comparison between different object detection techniques.

(1) untitled

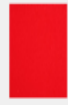

EAST
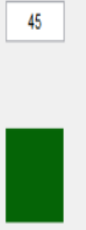

SOUTH

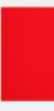

WEST

Figure 3 Traffic simulation - 1

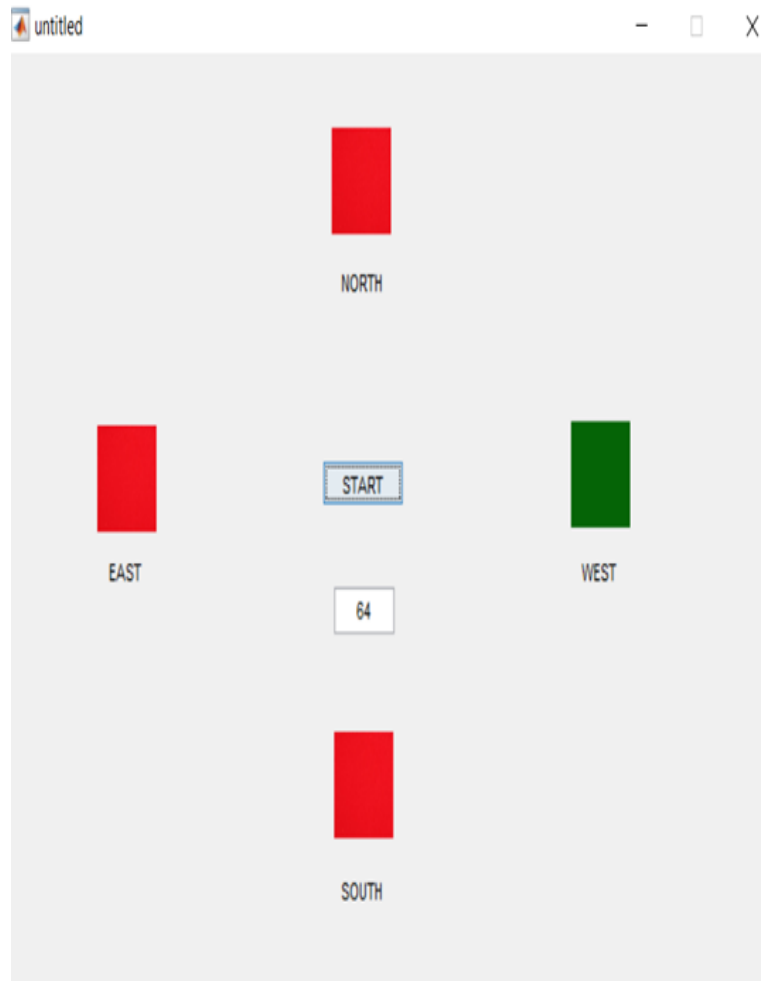

Figure 4 Traffic simulation - 2

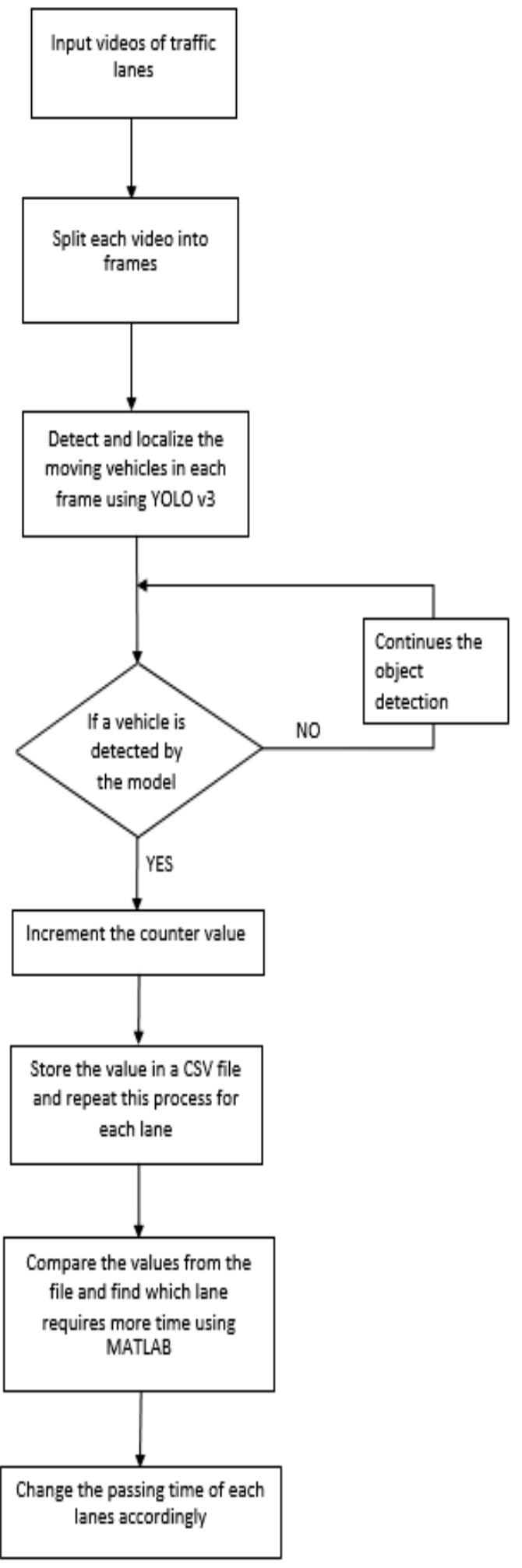

Figure 5 Working of traffic signal timing control 


\section{Results and discussion}

Table 1 Comparison between different object detection techniques

\begin{tabular}{llll}
\hline Hours(S) & Fixed time method & Q-learning method & Neural network \\
\hline 1 & 4121 & 4086 & 1067 \\
2 & 9232 & 8786 & 7232 \\
3 & 14233 & 11246 & 5348 \\
4 & 17952 & 16786 & 7854 \\
5 & 23414 & 22616 & 10641 \\
\hline
\end{tabular}

The traffic signal control management [2] are a bit less efficient than neural networks as described by them and considers traffic in only one road in a junction at a time, this may lead to congestion in other roads in the same road junction.

We use Yolo v3 in neural network for its accuracy in detecting smaller objects and it is convolutional for detecting vehicles in high traffic. YOLO v3 uses a variant of darknet, which has 53 layered networks trained on ImageNet and it makes detections at three different scales and therefore the output tensor will be 4 bounding box offsets, 1 object prediction and nearly 75 class predictions.

It works well for high traffic demands because it is not bounded by the curse of dimensionality and it is well suited for detecting objects in different resolutions and videos which have very less frames per second. This method will increase the accuracy by detecting vehicles of different sizes with utmost accuracy in video of lower resolutions.

Since our method is time wise dynamic too unlike Traffic Signal Control Management [2], it can be implemented for real time Traffic in urban areas where flow of traffic changes at any time and can be utilized within the standard traffic time (4 minutes). Which means we can manage traffic efficiently without increase or decrease in overall time for one traffic cycle.

\section{Conclusion and future work}

Traffic congestion has always been an issue when it comes to places of high population. Due to high population and inefficient traffic control methods, it is recorded that an average of 1274 accidents and 405 deaths occur every day across the country. Due to traffic congestion the roads cost around 60,000 crore every year including fuel wasted, productivity loss, air pollution and road accidents.
The traffic light waits and go timer set for 4 -way junctions are very inefficient due to the uneven vehicle density in each lane which changes with time.

This is a reason for many traffic violations and accidents.

Even though different techniques and methods are implemented to reduce these problems. Most of the methods are inefficient because traffic density is dynamic and changes with time. And the technique proposed will solve this problem and reduce the traffic congestion within the given standard traffic time by altering the go time in each lane according to the traffic density. The proposed system can be used in any traffic management solution towards real-time traffic density estimation and prediction.

Work still remains to be done in order to improve the computation time and efficient processing of video frames. Further enhancements are required in detecting vehicles. Multiple CCTV camera's fitted in different locations can be used for determination of the traffic density. Object detecting sensors can be used for detecting vehicles and determining traffic flow. In the future, with advanced monitoring technologies along with an improved deep learning algorithm we can easily determine traffic density more accurately and greatly reduced traffic congestion and accidents.

\section{Acknowledgment}

None.

\section{Conflicts of interest}

None.

\section{References}

[1] Zaidi AA, Kulcsár B, Wymeersch H. Back-pressure traffic signal control with fixed and adaptive routing for urban vehicular networks. IEEE Transactions on Intelligent Transportation Systems. 2016; 17(8):213443.

[2] Ghane S, Patel V, Mudliar K, Naik A. Using AI and machine learning techniques for traffic signal control 
Vishnu, Vikram et al.

management-review. International Journal of Engineering Research \& Technology.2017; 6(11):3368.

[3] Zhang R, Ishikawa A, Wang W, Striner B, Tonguz OK. Intelligent traffic signal control: Using reinforcement learning with partial detection. arXiv preprint arXiv:1807.01628. 2018.

[4] Wei H, Zheng G, Yao H, Li Z. Intellilight: a reinforcement learning approach for intelligent traffic light control. In proceedings of the SIGKDD international conference on knowledge discovery \& data mining 2018 (pp. 2496-505). ACM.

[5] Liang X, Du X, Wang G, Han Z. Deep reinforcement learning for traffic light control in vehicular networks. arXiv preprint arXiv:1803.11115. 2018.
[6] Gao J, Shen Y, Liu J, Ito M, Shiratori N. Adaptive traffic signal control: Deep reinforcement learning algorithm with experience replay and target network. arXiv preprint arXiv:1705.02755. 2017.

[7] Dheekonda RS, Panda SK, Khan N, Al-Hasan M, Anwar S. Object detection from a vehicle using deep learning network and future integration with multisensor fusion algorithm. SAE International in United States. 2017.

[8] Li J, Gu J, Huang Z, Wen J. Application research of improved YOLO V3 algorithm in PCB electronic component detection. Applied Sciences. 2019; 9(18):1-21

[9] Zhao ZQ, Zheng P, Xu ST, Wu X. Object detection with deep learning: a review. IEEE Transactions on Neural Networks and Learning Systems. 2019. 